\title{
MUESTRAS, VARIABILIDAD Y ERROR
}

\author{
JoRge DAgnino S. ${ }^{1}$
}

- Variable es cualquier atributo o característica que puede tomar diferentes valores de un set; una constante es aquella con un valor único invariable.

- Universo o población es el conjunto de todos los individuos que presentan una característica de interés susceptible de ser observada.

- Muestra es una parte de un universo a través de la cual se estudia una o varias características de interés.

- La variabilidad es la dispersión de los valores de una variable en una distribución teórica $o$ en una muestra. Puede ser conocida o desconocida y deriva de factores biológicos o de errores en la medición.

- Error es la diferencia entre el valor observado y el valor verdadero y puede ser aleatorio o sistemático (sesgo o bias).

- Los errores sistemáticos o sesgos son innumerables y pueden aparecer en cualquier parte del proceso de investigación y comunicación de los resultados. La defensa contra la variabilidad y el sesgo reside en muestras grandes, la estandarización, la asignación aleatoria y el uso apropiado del ciego o enmascaramiento.

La mayor parte de la investigación médica clínica está dirigida a observar diferencias entre grupos de personas con el objeto de identificar factores de riesgo o de pronóstico o, más frecuentemente, para averiguar si una determinada intervención produce mejores resultados que otra. Para averiguarlo, generalmente se efectúan, o debieran efectuar, los siguientes pasos secuenciales: formulación de una o varias hipótesis, formulación de los objetivos generales y específicos que se persiguen, diseño del experimento o ensayo para responder esas pregun- tas, realización de mediciones u observaciones con las que se obtienen datos, análisis de esos resultados y, finalmente, la interpretación y conclusiones. Los hallazgos son comunicados en ese mismo orden con el fin de que la comunidad médica pueda enterarse de esos resultados para guiar la práctica clínica. Idealmente, la descripción debe permitir replicar las condiciones experimentales con la mayor exactitud posible; si la descripción proporcionada no lo permite, entonces no cumple su propósito y se puede afirmar que es una mala descripción y, por ende, los resultados y conclusiones sólo pueden ser tomados como provisionales y no comprobados.

Aquí se presentan algunas consideraciones sobre conceptos fundamentales que muchas veces aparecen desperdigados en la literatura o sujetos a análisis técnico por lo que su significado no siempre es asido con facilidad por el médico clínico usuario ocasional de un texto de estadística. Por otro lado, diferentes definiciones y usos de los términos causan confusión. El riesgo es que se presente la información en forma demasiado elemental para muchos lectores que espero sabrán disculpar esto; para otros, esperamos, pueda ser una manera de precisar conceptos que estaban algo olvidados o confusos.

\section{VARIABLES versus CONSTANTES}

Una variables es cualquier atributo o característica que puede tomar diferentes valores de un set determinado; estos valores pueden ser números, caracteres o categorías. Cada variable tiene un nombre y un tipo de datos y es expresada en su medida específica: por ejemplo, peso corporal en kilogramos. Lo opuesto de una variable es una constante cuyo valor único es invariable.

En general se distinguen tres tipos de variables: independientes, dependientes y confundentes o de confusión. En la lectura crítica de un trabajo es indispensable identificar con claridad cada una de

Profesor Titular, División de Anestesiología, Pontificia Universidad Católica de Chile. 
ellas. La independiente es aquella que se manipula en el experimento o la que predice o determina el comportamiento de otra u otras variables; habitualmente son representadas por la letra " $x " \mathrm{y}$ se representan en el eje de las abscisas en un gráfico cartesiano. Las variables dependientes son aquellas en las que se evalúan los efectos; se designan con la letra "y" y se grafican en el eje de las ordenadas. Las variables confundentes son aquellas ajenas a la relación que se estudia pero que podrían ejercer un efecto sistemático sobre los resultados; controlar estas variables confundentes es función principal del diseño del trabajo.

\section{MUESTRA Y UNIVERSO}

La principal diferencia entre la práctica clínica y la investigación médica reside en su proyección. En ambas se recolectan datos de individuos pero sólo en la investigación se pretende hacer una generalización de lo encontrado en el grupo estudiado a un grupo más grande de individuos con características similares: usar la información de una muestra para hacer una inferencia sobre una población más amplia. El concepto de muestra y universo o población a veces se presta a confusión porque su sentido exacto se olvidó o por que, por lo mismo, en ocasiones se usa laxamente.

1) Universo o población es el conjunto de todos los individuos que presentan una o varias características de interés susceptibles de ser observadas. Un universo puede ser virtualmente ilimitado, cuando su extensión exacta no puede ser determinada (por ejemplo, la humanidad, una determinada especie animal o vegetal), o bien limitado, cuando sí se puede determinar (los habitantes de un determinado país o ciudad). Un universo pequeño puede ser estudiado en su totalidad y en ese caso la estadística se usa para resumir y describir los hallazgos. Muchas veces se usa, erróneamente, población para referirse a muestra. Un universo está definido por parámetros que son cantidades que definen un modelo teórico; por ejemplo, una distribución normal está definida por sus parámetros, media y desviación estándar. Los parámetros no deben confundirse con variables que son cantidades obtenidas a través de mediciones y que varían entre individuos o en un mismo individuo a lo largo del tiempo.

2) Muestra, es una parte de un universo a través de la cual se estudian sus características. Las razones para hacerlo son de índole práctica o económica, cuando estudiar a toda la población de inte- rés podría tomar demasiado tiempo y recursos. $\mathrm{La}$ muestra debe ser obtenida de tal manera que los resultados conseguidos en su observación o medida puedan ser generalizados al universo mediante un proceso lógico de inducción. La manera en que se obtiene la muestra es crucial en la posibilidad de hacer esa generalización; en efecto, los errores en la elección de la muestra son unos de los más frecuentes y fatales en la literatura médica.

\section{VARIABILIDAD}

Es un hecho fácilmente constatable que en una población o muestra cualquiera, la variabilidad en las características y en las respuestas es la norma. La variabilidad es una medida de la dispersión de los datos en una distribución, sea esta teórica o de una muestra; medidas de variabilidad son la varianza, la desviación estándar, cuartiles o deciles, y rango. Se puede decir que la estadística se ocupa fundamentalmente de la variabilidad y de la estimación de sus efectos en los resultados obtenidos; esto subraya la necesidad de enseñar sobre variabilidad para enseñar el "pensamiento estadístico". En las pruebas de hipótesis, la diferencia entre los grupos se ubica en el numerador de las fórmulas y la variabilidad en el denominador; esto quiere decir que para una determinada diferencia, la magnitud del estadístico calculado con la prueba es inversamente proporcional a la variabilidad y por ende la $\mathrm{p}$, e igual cosa sucede con los intervalos de confianza, es mayor mientras mayor es la variabilidad.

La variabilidad puede ser biológica, por diferencias entre los individuos, o bien diferencias en un mismo individuo a lo largo del tiempo, como resultado de cambios de corto plazo o infradianos (presión arterial o frecuencia del pulso), o en períodos más largos (circadianos o supradianos). Los ejemplos son innumerables en torno a características físicas, fisiológicas y psicológicas: color del pelo o estatura, metabolismo basal o natremia, carácter o personalidad.

La variabilidad puede también provenir de errores en la medición de las características de interés, donde error es la diferencia entre el valor observado y el valor verdadero. Estos errores pueden ser conocidos, incluso cuantificados, o desconocidos, y pueden derivar de los métodos o instrumentos de medición, depender del observador u observadores, y de la variabilidad de la característica observada.

Los errores pueden ser aleatorios o sistemáticos. Error aleatorio es aquella parte de nuestra experiencia que no podemos predecir; estadísticamente, es la variabilidad muestral; lo inverso de error 
aleatorio es la precisión. Error sistemático o sesgo (bias en inglés) se debe a todo lo que no es variabilidad muestral; lo inverso de sesgo es la validez. Aumentar el número de observaciones o su repetición reduce el error aleatorio, cuya dirección no es predecible, pero no sucede lo mismo con el error sistemático, cuya dirección es en un solo sentido. Si se conoce la dimensión del error sistemático es posible reducir o eliminar su impacto mediante ajustes, cosa que no es factible en el caso del error aleatorio.

Las fuentes o causas de error aleatorio o sistemático son innumerables y algunas de ellas se citan aquí a modo de ejemplo, pues los trabajos de investigación deben dar cuenta en su descripción de cómo se intentó controlar esta variabilidad, cuando esto es factible, o cómo se aseguró que afecte por igual a todos los grupos de estudio. Esto es particularmente importante para el error sistemático por lo que se le dedica una sección aparte más abajo. Son fuentes de error:

1. Variabilidad de los sujetos (inter e intra). Causas: fluctuaciones fisiológicas o patológicas (infradianas, circadianas y supradianas); variaciones en la motivación, esfuerzo o cumplimiento de las instrucciones; accidentes y equivocaciones; interacciones con observadores; efectos del medio ambiente; variabilidad de la intervención (por ejemplo, diferentes partidas de medicamentos con diferentes efectos; diferentes operadores con diferentes capacidades); existencia de comorbilidades.

2. Variabilidad dependiente de los observadores (inter e intra): puede ser disminuida por la existencia de guías claras, entrenamiento previo o chequeos. Fuentes: tiempo y esfuerzo; entrenamiento y experiencia; fatiga física o psicológica; variaciones del medio ambiente; interacción con sujetos; accidentes y equivocaciones.

3. Variabilidad dependiente de los instrumentos (inter e intra). Error intrínseco del instrumento de medición: mal diseño o funcionamiento; problemas de calibración, mantención y desgaste; error de procesamiento; efectos del medio ambiente o circunstancias de la medición.

\section{ERRROR SISTEMÁTICO O SESGO}

Sackett define sesgo o bias como "cualquier proceso en cualquier etapa de la inferencia que tiende a producir resultados o conclusiones que difieren sistemáticamente de la verdad". Hace más de veinte años intentó un catálogo de sesgos; si bien descritos en el ámbito de casos versus controles, su diversidad y abundancia son reflejo de la ubicuidad y subrepción con que se pueden colar en cualquier investigación y en cualquier etapa de ella. Subrayan la necesidad de tomar todas las precauciones para evitarlos y la de los lectores que deben estar siempre atentos a su posibilidad. El diseño del trabajo es el plan que busca asegurar que en su ejecución se despejarán los errores y las confusiones derivadas de los sujetos, del medio ambiente o del transcurso del tiempo.

En todo trabajo existe cierto grado de sesgo y es prácticamente imposible eliminarlos del todo. Por lo tanto, es aún más importante que el lector pueda contar con la mayor precisión y completitud posible la información de lo que se hizo y de lo que se encontró. Sólo así podrá hacer un juicio informado sobre el grado de influencia que esos sesgos pueden tener sobre las conclusiones del trabajo.

Los sesgos aparecen en todas las etapas de una investigación: en la planificación y el diseño del estudio, en su ejecución, el análisis de los resultados, las conclusiones y en la comunicación y publicación. Estos últimos, particularmente la omisión de información, inadvertida o premeditada, son particularmente nocivos.

Se han descrito numerosísimas formas de sesgo y aun así pareciera que el catálogo no es completo. La terminología, además, puede ser confusa, sobrepuesta y con connotaciones específicas a cada especialidad o tipo de estudio. Una lista incompleta podría incluir errores de concepto, diseño, selección, instrucción, diagnóstico, oportunidad, evaluación, observación, instrumento, recuerdo, respuesta, seguimiento, registro, análisis, interpretación, comunicación y de publicación. Algunos sesgos tienen nombres propios como el de Hawthorne o Berkson. Los sesgos de publicación han recibido particular atención y generado gran controversia poniendo en duda incluso una proporción importante de los hallazgos publicados. Ello ha llevado a diversas estrategias como el registro de ensayos clínicos y la mayor transparencia en los datos obtenidos llevando a aquello de "confiar pero verificar". De esto se trata por lo demás la lectura crítica como resalta Lehman con su Nullius in Verba, el lema fundacional de la Royal Society a mediados del siglo XVII.

La defensa contra la variabilidad y el sesgo reside en muestras grandes, la estandarización, la asignación aleatoria y el uso apropiado del ciego o enmascaramiento. Es por ello que se debe desconfiar intuitivamente $o$, por lo menos tomar con escepticismo, los resultados conseguidos con muestras pequeñas, aquellos con definiciones operacionales insuficientes o imprecisas, y aquellos en que no se 
puede juzgar la calidad de la asignación aleatoria o el enmascaramiento.

\section{CIEGO O ENMASCARAMIENTO}

El término enmascaramiento o ciego significa mantener a los sujetos del estudio, a los investigadores, asesores y evaluadores sin conocimiento del grupo asignado y, ojalá, de la intervención hecha. Si bien en menor medida que con el sesgo, los conceptos relativos al enmascaramiento no son bien comprendidos, usados, ni bien descritos en la literatura. Para que el lector tenga la posibilidad de discernir si se usó adecuadamente, el enmascaramiento debe ser descrito con mayor precisión que el simple "se usó un ciego simple" o un "doble ciego".

\section{ASIGNACIÓN ALEATORIA}

En estadística, el término aleatorio (random en inglés) tiene un significado técnico preciso: cada sujeto tiene una probabilidad conocida, generalmente idéntica, de ser incluso en la muestra o de ser asignado a uno u otro grupo. La manera más básica es la asignación aleatoria (randomisation) simple basada en tablas de números aleatorios o en un generador computacional de ellos. Otras alternativas dependientes del diseño del estudio incluyen la asignación en bloque, para asegurar un número similar en cada grupo, o la asignación estratificada, para balancear en cada grupo alguna característica de interés. Tampoco basta aquí describir en un trabajo con un simple "se asignó aleatoriamente"; debe describirse claramente el método de asignación aleatoria.

\section{REFERENCIAS}

1. Altman DG, Bland JM. How to randomise. BMJ 1999; 319: 703.

2. Altman DG, Bland JM. Treatment allocation in controlled trials: why randomise? BMJ 1999; 318: 1209.

3. Altman DG, Bland JM. Variables and parameters. BMJ 1999; 318: 1667.

4. Altman DG. Practical Statistics for Medical Research. London: Chapman \& Hall, 1991.

5. Bland M. An Introduction to Medical Statistics. 3rd Ed, Oxford: OUP, 2006.

6. Califf RM, McCall J, Harrington RA. Assessing Research Results in the Medical Literature. Trust but Verify. JAMA 2013; 173: 10531055.

7. Chin R, Lee BY. Principles and Practice of Clinical Trial Medicine. Amsterdam: Academic Press, 2008.

8. Dawson-Saunders B, Trapp RG.
Bioestadística médica. México D.F: Manual Moderno, 1993.

9. Day SJ, Altman DG. Blinding in clinical trials and other studies. BMJ 2000; 321: 504.

10. Farrugia P, Petrisor BA, Farrokhyar F, Bhandari M. Research questions, hypothesis and objectives. Can J Surg 2010; 53: 278-281.

11. Gould R. Variability: one statistician's view. Statistics Education Research J 2004; 3: 7-16.

12. Hopewell S, Loudon K, Clarke MJ, et al. Publication bias in clinical trials due to statistical significance or direction of trial results (Review). The Cochrane Collaboration, London: John Wiley \& Sons, 2009.

13. Lehman RS. Nullius In Verba. Don't Take Anyone's Word for It. JAMA 2013; 173: 1049-1050.

14. Pannucci CJ, Wilkins EG. Identifying and Avoiding Bias in Research. Plast Reconstr Surg 2010; 126: 619-625.
15. Portney LG, Watkins MP. Foundations of Clinical Research. Applications to practice. 2nd ed. Upper Saddle River: Prentice-Hall, 2000

16. Sackett DL. Bias in analytic research. J Chronic Dis 1979; 32: 51-63.

17. Schulz KF, Chalmers I, Altman DG. The Landscape and Lexicon of Blinding in Randomized Trials. Ann Intern Med 2002; 136: 254259.

18. Schulz KF, Grimes DA. Blinding in randomised trials: hiding who got what. Lancet 2002; 359: 696-700.

19. Sica GT. Bias in Research Studies. Radiology 2006; 238: 780-789.

20. Snee RD. Statistical Thinking and Its Contribution to Total Quality. The American Statistician 1990; 44: 116-121.

21. Wild CJ, Pfannkuch. Statistical Thinking in Empirical Enquiry. International Statistical Review 1999; 67: 223-265.
Correspondencia a:

Dr. Jorge Dagnino S.

jdagnino@med.puc.cl 